Mini Review

\title{
Post procedure urinary tract infection after urodynamic
}

\begin{abstract}
Introduction: Urodynamic pressure flow study is invasive investigation to assess lower urinary tract symptoms. We evaluated risk of UTI after the procedure.

Patients and methods: We conducted prospective evaluation of patients coming for urodynamic study. Patients with symptomatic and dipstick test positive UTI were excluded. Patients were assessed by post procedure MSU and symptom questionnaire.
\end{abstract}

Results: Rate of infection was $5.6 \%$ with no significant relationship to other parameters.

Conclusion: Data is comparable and routine antibiotics are not recommended.
Volume 5 Issue 4 - 2017

\author{
Mohammad Hassan Khan, S Knight, O Baldo, \\ P Koenig, N Shaikh \\ Department of Urology, Airedale General Hospital NHS \\ Foundation Trust, UK
}

\author{
Correspondence: Mohammad Hassan Khan, Department \\ of Urology, Airedale General Hospital NHS Foundation Trust. \\ Skipton road, Keighley, BD20 6TD, UK, \\ Email drtahirkheli@doctors.org.uk
}

Received: August 03, 2017 | Published: October 09, 2017

\section{Introduction}

Urodynamic pressure flow studies (PFS) are increasingly utilized in assessing patients with lower urinary tract (LUT) dysfunction. ${ }^{1-3}$ This procedure is not without risks, and urinary tract infection (UTI) is the commonest side effect. ${ }^{4-6}$ This is because of instrumentation of lower urinary tract. The signs and symptoms of this morbidity are haematuria, dysuria and increased frequency of micturition. ${ }^{7}$ The published incidence of urinary tract infection after urodynamic study is surprisingly high, running between $2.1 \%$ to $21 \% .^{1-5}$ To evaluate the incidence of UTI and future need for prophylactic antibiotic we conducted this audit in our unit.

\section{Patients and methods}

This audit is a prospective evaluation of consecutive 107 patients who attended our urodynamic department. A pretest assessment was completed for all patients recording;

i. Indications for procedure.

ii. History of recurrent UTI's.

iii. History of diabetes mellitus.

iv. Post Micturition residual volume.

v. Pre-procedure Urine dipsticks.

After that Urodynamic study was performed under strict aseptic techniques and hospital guidelines. After the procedure patients were asked to submit a Mid-Stream Urine specimen 2 days after the urodynamic study.

They were also requested to fill in a questionnaire asking;

I. If they had urinary tract infection after procedure?

II. If they were prescribed any antibiotics treatment?

They were advised to fill the questionnaire and send it back to Urology department 1 week after the procedure. Patients were provided with self-addressed and stamped envelope to send back the questionnaire. One hundred and thirty patients agreed to take part and we obtained complete data on 107 patients. MSU culture results confirming UTI were faxed to General Practitioners as they were received from microbiology department for patient assessment and antibiotics prescribing in primary care. Those results were faxed as high priority and also an urgent letter was sent.

\section{Results}

Out of 107, there were $61(57 \%)$ Female and $46(43 \%)$ were male patients (Figure 1). Median age of patients was 61 years. Fifteen (14\%) patients had symptoms of dysuria and frequency after the procedure suggestive of urinary tract infection or symptoms secondary to catheterisation. Out of those 15 patients 9 were female and 6 male patients. $6(5.6 \%)$ patients out of those who had symptoms of UTI had diagnosis confirmed on Dipstick test and Mid-Stream Urine culture and sensitivity. $9(9.6 \%)$ patients settled after 2-3days without any treatment and were not classed as symptomatic UTI because of normal dipstick test and no growth on MSU. The patients with confirmed UTI (5.6\%) needed antibiotics to relieve their symptoms.

There was no significant relationship to

a. Indication.

a. History of Diabetes Mellitus.

b. History of recurrent UTI's.

c. Pre/posttest residual volumes.

d. Pretest urine Dipstick.

In patients who had confirmed urinary tract infections 3-culture specimen grown Escherichia Coli and 3 specimens grew mixed growth of organisms. Their symptoms settled after course of antibiotics.

\section{Discussion}

In our Urodynamic department a senior physiotherapist conducts urodynamic Study. She is also responsible for Uro-therapy and pelvic floor evaluation and management of pelvic floor dysfunction. Responsible Urology consultants conduct weekly Multidisciplinary meetings to discuss difficult cases and management planning. Urodynamic investigation being an invasive test is not without any side effects and complication., ${ }^{7,8}$ Urinary tract infection is 
common complication. ${ }^{4-6}$ An incidence of UTI in a patient attending Urodynamic study triggered the need for auditing and making a safe pathway in our department. A prospective study was arranged after departmental meeting with infection control team to find out rate of UTI after Urodynamic study. This audit study was registered and approved with audit department.

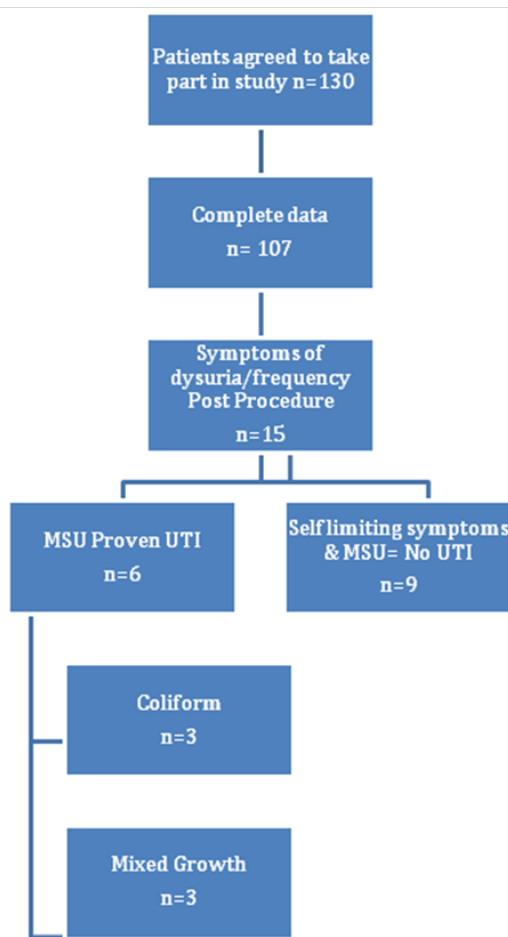

Figure I Results.

UTI is second commonest cause of bacterial infection in Europe, ${ }^{9,10}$ and has financial implications, which are annually estimated to 1.6 billion US $\$$ around the world., ${ }^{411,12}$ A pre-test Urinary tract infection diagnosed clinically or on dipsticks was exclusion criteria for the test. These patients were treated for UTI and procedure re-scheduled after 1-2 weeks to get proper results from the study. In literature there was varied risk of UTI after the procedure. The published literature has shown high UTI risk in patients with high residual volume, diabetes and advance age and discussed prophylactic antibiotics before the procedure in high-risk patients..$^{4,13-15}$ In our study rate of MSU confirmed UTI is $5.6 \%$, which is comparable to published literature. Recent work by M. Nadeem et al from Kent, UK suggested high incidence of UTI of $12 \%$ and advised antibiotics prophylaxis for highrisk patients. ${ }^{4}$

In our department we strictly follow aseptic techniques and regular visits from Hospital infection control advisers help us to anticipate any problems. Following the Antimicrobial stewardship program's advice we aim to prevent and treat infections while keeping the check on misuse of antibiotics. ${ }^{16-20}$ We have developed a protocol for the patient attending urodynamic. We do not routinely use antibiotics prophylaxis. In high-risk patients (Catheter in-situ/Intermittent self-catheterization and history of recurrent UTI's) we do consider antibiotic prophylaxis.

\section{Conclusion}

We recorded an incidence of $5.6 \%$ UTI after urodynamics (Coliform 3 patients $=2.8 \%$, Mixed growth 3 patients $=2.8 \%$ ). This incidence occurred without any antibiotic prophylaxis. Our results are better than recently published data. ${ }^{4}$ Overall urodynamic study is a safe and well-tolerated procedure with a low incidence of UTI. We therefore do not recommend routine antibiotic prophylaxis. To prevent Urinary tract infections we recommended;

I. Cancelling and re scheduling procedure if patient has active UTI.

II. Urine Dipstick tests before all procedures.

III. Complete aseptic insertion of Bladder catheter.

IV. Use of sterile gloves by operator.

V. Use of single use, disposable sterile equipment e.g. catheters connectors, tubing and diaphragms in Urodynamic setup.

VI. Use of new saline bag for every patient.

VII. Antibiotic prophylaxis for patients with long-term catheter, intermittent self-catheterization and history of recurrent UTI's.

\section{Conflicts of interest}

Authors declared there are no conflicts of interest.

\section{Acknowledgements}

None.

\section{Funding}

None.

\section{References}

1. Quek P, Tay LH. Morbidity and significant bacteriuria after urodynamics studies. Ann Acad Med Singapore. 2004;33:754-757.

2. Yip SK, Fung K, Pang MW, et al. A study of female urinary tract infection caused by urodynamic investigations. Am J Obstet Gynecol. 2004;190:1234-1240

3. Harari D, Malone-Lee J, Ridgway GL. An Age-related Investigation of Urinary Tract Symptoms and Infection following Urodynamic Studies. Oxford J/Med/age and ageing. 1994;23(1):62-64.

4. Nadeem M, Sheikh MI, Sait MS, et al. Is urinary tract infection after urodynamic study predictable? Urological Science. 2017;1-3.

5. Onur R, Ozden M, Orhan I, et al. Incidence of bacteraemia after urodynamic study. J Hosp Infect. 2004;57:241-244.

6. Sabanathan K, Duffin HM, Castleden CM. Urinary tract infection after cystometry. Age Ageing. 1985;14(5):291-295.

7. Lowder JL, Burrows LJ, Howden NL, et al. Prophylactic antibiotics after urodynamics in women:a decision analysis. Int Urogynecol J. 2007;18(2):159-164.

8. Sabanathan K, Duffin HM, Castleden CM. Urinary tract infection after cystometry. Age Ageing. 1985;4:291-295.

9. Xia J, Gao J, Tang W. Nosocomial infection and its molecular mechanisms of antibiotic resistance. Biosci Trends. 2016;10(1):14-21.

10. M André, J Ahlqvist-Rastad. Lower urinary tract infection (UTI) in women - Treatment recommendation. Sweden: The Med Prod Agency; 2007. pp. 18.

11. B Foxman. Epidemiology of urinary tract infections:incidence, morbidity, and economic costs. Am J Med. 2002;113:5S-13S.

12. Cardwell SM, Crandon JL, Nicolau DP, et al. Epidemiology and economics of adult patients hospitalized with urinary tract infections. Hosp Pract. 2016;44 (1):33-40. 
13. Klingler HC, Madersbacher S, Djavan B, et al. Morbidity of the evaluation of the lower urinary tract with transurethral multichannel pressure-flow studies. J Urol. 1998;159(1):191-194.

14. James R, Hijaz A. Lower urinary tract symptoms in women with diabetes mellitus: a current review. Curr Urol Rep. 2014;15(10):440.

15. Bombieri L, Dance DA, Rienhardt GW, et al. Urinary tract infection after urodynamic studies in women: incidence and natural history. $B J U$ In. 1999; 83(4):392-395.

16. Allerberger F, Gareis R, Jindrák V, et al. Antibiotic stewardship implementation in the EU: the way forward. Expert Rev Anti Infect The. 2009;7(10):1175-1183.
17. Lesprit P, Brun-Buisson C. Hospital antibiotic stewardship. Curr Opin Infect Dis. 2008;21(4):344-349.

18. Cefai C. NICE Guideline: Antimicrobial stewardship:systems and processes for effective antimicrobial medicine use. 2015.

19. Dohnhammar U. SWEDERS 2010, A report on Swedish antibiotic utilisation and resistance in human medicine. Sweden; 2010. p. 1-50.

20. Nilholm H, Holmstrand L, Ahl J, et al. An Audit-Based, Infectious Disease Specialist-Guided Antimicrobial Stewardship Program Profoundly Reduced Antibiotic Use Without Negatively Affecting Patient Outcomes. Open Forum Infect Dis. 2015;2(2):ofv042. 